\title{
TITLE:
}

\section{$<$ News> Drs. Jane Goodall and Toshisada Nishida win 2008 Leakey Prize!}

$\operatorname{AUTHOR}(S)$ :

PAN Editors

CITATION:

PAN Editors. <News> Drs. Jane Goodall and Toshisada Nishida win 2008 Leakey Prize!. Pan Africa News 2008, 15(1): 1-2

ISSUE DATE:

2008-06

URL:

http://hdl.handle.net/2433/143491

RIGHT:

Copyright (C) Pan Africa News. 


\section{<NEWS>}

\section{Drs. Jane Goodall and Toshisada Nishida win 2008 Leakey Prize!}

The Leakey Foundation will award 2008 L.S.B. Leakey Foundation Prize for Multidisciplinary Research on Ape and Human Evolution to two distinguished chimpanzee research pioneers, Dr. Jane Goodall and Dr. Toshisada Nishida ( $\$ 25,000$ each) during the events from October 30 to November 1, 2008 at the California Academy of Sciences in San Francisco USA, commemorating the 40th anniversary of the establishment of the world's most successful private funder of human origins science. On October 31, public 
lectures will be given by Drs. Goodall and Nishida.

On November 1, 2008, Scientific Symposium and Public Forum for Primatology will take place featuring a panel of six world renowned primatologists led by the two Leakey Prize laureates. The topic will be "What does it mean to be a primate: a human discussion." That evening, Leakey Prize Gala and Award Ceremony will be held honoring Drs. Goodall and Nishida.

This prize is awarded to prominent anthropologists for their contributions to multidisciplinary studies on human origins. The first winner was Dr. Philip Tobias in 1991 the second was Dr. Desmond Clarke in 1996, and the third was Dr. Frances Clarke-Howell in 1998.

Dr. Jane Goodall, guided by her mentor, the late Dr. Louis Leakey, initiated the longest field study of wild chimpanzees at Gombe on the shore of Lake Tanganyika, East Africa in 1960. In her first year at Gombe, she observed some epoch-making facts that surprised anthropologists of the time. She found that chimpanzees strip leaves off twigs to make tools for fishing termites from termite mounds. On hearing the news, Dr. Leakey left a well-known comment, "Now we must redefine tool, redefine man, or accept chimpanzees as humans.” She also observed chimps hunting and eating medium-sized mammals for the first time, proving that humans are not the only meat-eating apes. Dr. Goodall has been the most famous, legendary zoologist and her comprehensive book "The Chimpanzees of Gombe: Patterns of Behavior" (1986) has been the chimpanzee researcher's bible for more than 20 years.

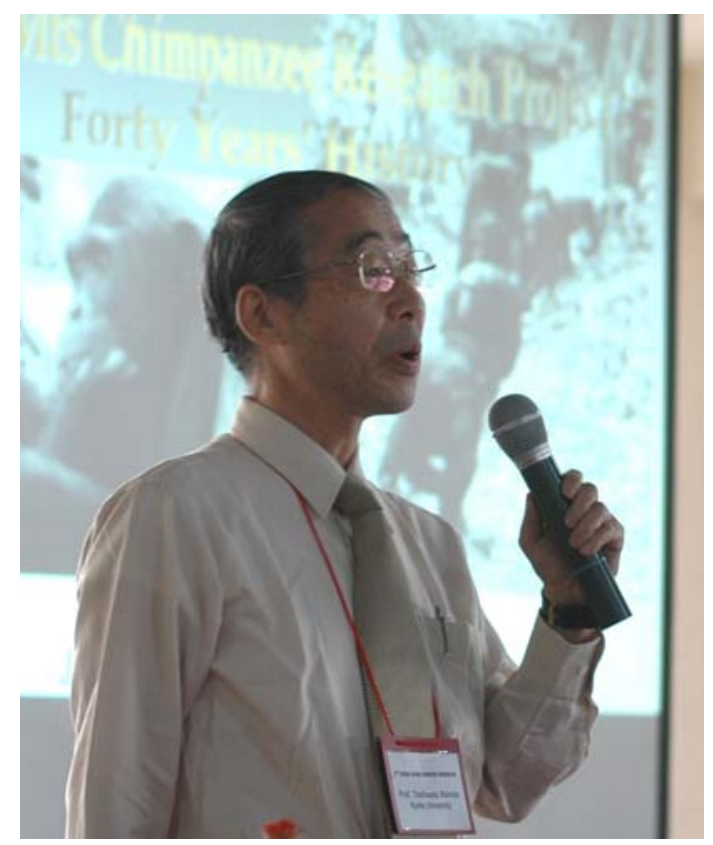

Dr. Toshisada Nishida
Dr. Toshisada Nishida, when he was a graduate student, joined the Kyoto University's Ape Expedition to Africa organized by Drs. Kinji Imanishi and Junichiro Itani. In 1965, he succeeded in establishing the second longest running wild chimpanzee research site at the Mahale Mountains on the shore of Lake Tanganyika, about $100 \mathrm{~km}$ south of Gombe. Since then, in collaboration with many researchers such as the late Drs. Kenji Kawanaka and Shigeo Uehara, Dr. Nishida has persistently explored all aspects of our closest kin in their natural habitats. Initial important discoveries included the finding of definite social structure he called 'unit group' (or community) and female transfer system between chimpanzee unit groups, a sharp contrast to many female-bonded primate societies. He has also revealed the hostile intergroup relationships, male alliance strategies, and cultural behaviors of chimpanzees. 